\title{
Análise Numérica do Comportamento de Elementos de Madeira em Situação de Incêndio
}

\author{
Julio Cesar Molina ${ }^{1}$, Carlito Calil Junior ${ }^{1}$, Érica Fernanda Aiko Kimura ${ }^{1}$, \\ Edna Moura Pinto ${ }^{2}$, Ronaldo Regobello ${ }^{3}$ \\ ${ }^{1}$ Departamento de Engenharia de Estruturas, Escola de Engenharia de São Carlos - EESC, \\ Universidade de São Paulo - USP \\ ${ }^{2}$ Centro de Tecnologia, Universidade Federal do Rio Grande do Norte - UFRN \\ ${ }^{3}$ Universidade Tecnológica Federal do Paraná - UTFPR
}

\begin{abstract}
RESUMO
A principal característica da ação térmica que age sobre uma estrutura, que aparece em resposta às temperaturas elevadas, é a curva que fornece a temperatura dos gases em função do tempo de incêndio (TRRF). A partir do tempo de incêndio, considerando curvas padronizadas internacionais, obtém-se a temperatura fictícia dos gases e, por esta temperatura, determinase a temperatura no elemento estrutural analisado. A maioria dos modelos numéricos propõe métodos simplificados de análise. O objetivo deste trabalho de pesquisa foi o estudo numérico de elementos estruturais de madeira em altas temperaturas com seção transversal $6 \times 16 \mathrm{~cm}$, a partir da utilização do software ANSYS. São apresentados os principais elementos utilizados na modelagem numérica do sistema e também a comparação com resultados experimentais. Os resultados numéricos apresentaram uma boa concordância com os resultados experimentais.
\end{abstract}

Palavras-chave: elementos estruturais de madeira, modelagem numérica, resistência ao fogo.

\section{Numerical Analysis of the Behavior of Timber Elements under Fire Conditions}

\begin{abstract}
The main characteristic of the thermal action on a structure, which appears in response to high temperatures, is a curve that shows gas temperature versus time of fire (TRRF). Based on the time of fire, considering international standard curves, it is possible to obtain the temperature of the fictitious gases in the environment and, through this temperature it is possible to determine the temperature in the analyzed structural element. Most numerical models propose simplified methods of analysis. The purpose of this research was the numerical study of structural timber elements with $6 \times 16 \mathrm{~cm}$ cross section under fire conditions. The computational code ANSYS was used in the performance of the numerical analysis. The main elements used in the numeric modeling of the system were presented, as well as the comparison with the experimental results. The numerical results showed good concordance with the experimental results.
\end{abstract}

Keywords: structural elements of wood, numerical model, fire resistance. 


\section{INTRODUÇÃO}

O tema "Análise das Estruturas em situação de incêndio" vem ganhando cada vez mais espaço no cenário nacional. Neste contexto, a Escola de Engenharia de São Carlos, a Escola Politécnica, ambas da USP, e a Escola de Engenharia da UNICAMP, reunidas no Projeto Temático FAPESP, "Segurança das Estruturas em Situação de Incêndio", vêm impulsionando as pesquisas referentes à análise termoestrutural de elementos estruturais de concreto, aço e também de madeira, além de estruturas mistas de aço-concreto e de madeiraconcreto. Um dos principais objetivos deste Projeto Temático foi, inicialmente, a construção do "primeiro forno horizontal de ensaios" no Brasil, apto a avaliar vigas e lajes, em escala natural, sob carregamento e em situação de incêndio. O referido forno encontra-se instalado na EESC/USP e, atualmente, está em fase de finalização, sendo que a sua conclusão está prevista para o primeiro semestre de 2012. Vale mencionar que, atualmente, somente a UNICAMP possui um forno vertical para o ensaio de pilares. Os laboratórios de Furnas-GO e do IPTSP possuem fornos para o ensaio de paredes. Por motivos de ordem econômica e, principalmente, pela inexistência de equipamentos dessa magnitude para a realização de ensaios de elementos estruturais, grande parte das pesquisas no Brasil, relacionadas à análise das estruturas em situação de incêndio, tem tido caráter essencialmente numérico. Além disso, grande parte dos estudos numéricos refere-se à análise de elementos de aço e concreto.

A resistência ao fogo é definida como a capacidade de um material ou elemento estrutural permanecer, durante um tempo determinado, exercendo as funções para as quais foi projetado, sob a ação de incêndio. No caso da madeira, quando submetida às altas temperaturas, sofre degradação por combustão resultando na formação de carvão que protege suas camadas inferiores. Como consequência, ocorre a diminuição das propriedades de resistência e rigidez da madeira aquecida sob a camada carbonizada e a redução da seção resistente do elemento estrutural devido a formação de carvão. O texto de revisão da ABNT NBR 7190 (1997) traz recomendações sobre o dimensionamento de peças de madeira em altas temperaturas.
As chamadas curvas padronizadas ou curvaspadrão têm sido amplamente adotadas na realização de ensaios de elementos estruturais em altas temperaturas. A partir do tempo de incêndio, conhecido no meio técnico como "Tempo Requerido de Resistência ao Fogo" (TRRF), é possível obter na curva de incêndio-padrão, a temperatura fictícia dos gases do ambiente e, por meio dessa temperatura, uma temperatura no elemento estrutural que será usada no seu dimensionamento. As curvas padronizadas foram definidas por consenso pelos meios técnico e científico, sendo que as curvas mais utilizadas, neste caso, são a ISO 834 (1999) e a ASTM E-119 (2000). Essas duas curvas apresentam comportamentos muito semelhantes entre si (Figura 1).

A principal diferença entre uma curva natural e uma curva de incêndio-padrão é o fato de a primeira possuir um ramo ascendente (fase de aquecimento) e um ramo descendente (fase de resfriamento), enquanto a segunda não considera $\mathrm{o}$ ramo descendente da curva. A ocorrência da elevação da temperatura em elementos estruturais, em decorrência de incêndio, implica na necessidade de se conhecer as propriedades térmicas e mecânicas dos materiais empregados no elemento estrutural. Por outro lado, para a determinação das ações térmicas nestes elementos é necessário o conhecimento dos mecanismos pelo qual o calor é gerado no ambiente e, consequentemente, o modo como ele é propagado no seu interior. A ação térmica é proveniente de um fluxo de calor, o qual consiste de uma parcela de radiação e outra de convecção, que, por sua vez,

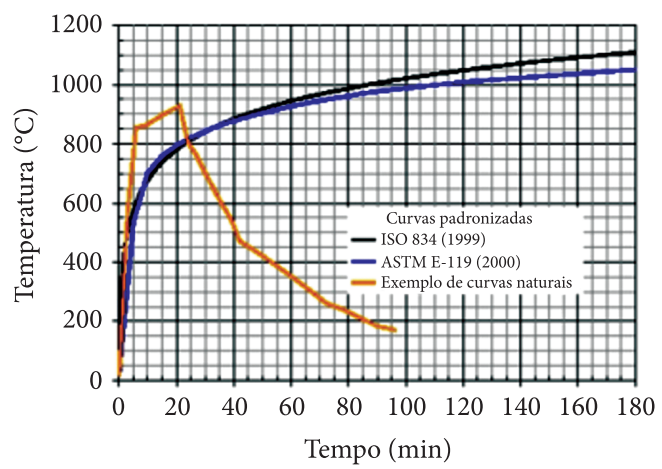

Figura 1. Curvas de incêndio-padrão e curva natural de incêndio. Fonte: Costa \& Silva (2002).

Figure 1. Standard-curves and curve of natural fire. Source: Costa \& Silva (2002). 
provoca o aumento da temperatura na estrutura em função do coeficiente de transferência de calor.

$\mathrm{Na}$ determinação do fluxo de calor radiante, é necessário o conhecimento da constante de StefanBoltzmann, além dos parâmetros relacionados às emissividades do material, que quantificam a energia radiante emitida pela superfície do material analisado. Existem basicamente dois tipos de modelos para a análise de estruturas em situação de incêndio, que são os modelos simplificados e os modelos avançados de cálculo. Os modelos simplificados permitem a obtenção da elevação da temperatura de modo homogêneo para toda a seção transversal e ao longo do comprimento de interesse do elemento estrutural, por meio de equações analíticas. Por outro lado, os modelos avançados têm como base os métodos numéricos e sua utilização depende do uso de microcomputadores para a resolução do problema.

O objetivo deste trabalho consistiu na avaliação numérica, através do software ANSYS, da evolução da temperatura a 10 e $20 \mathrm{~mm}$ de profundidade, numa seção transversal de madeira, de eucalipto citriodora, com dimensões $6 \times 16 \mathrm{~cm}$, submetida ao incêndio-padrão, com aquecimento dado de acordo com a curva proposta pela norma ISO 834 (1999).

\section{MATERIAL E MÉTODOS}

O presente trabalho teve origem a partir da necessidade da obtenção de informações a respeito da modelagem de vigas mistas de madeira e concreto. Optou-se então, como uma pré-análise do sistema misto, pelo estudo independente dos materiais madeira e concreto, submetidos a altas temperaturas. Os resultados da análise numérica do elemento de madeira de eucalipto citriodora considerado com seção transversal de $6 \times 16 \mathrm{~cm}$ foram comparados com os resultados experimentais obtidos por Regobello et al. (2006) e Pinto (2005). No processamento numérico do sistema avaliado no presente trabalho, considerou-se a curva padronizada de aquecimento ISO 834 (1999). Vale mencionar ainda que, segundo Regobello et al. (2006), para temperaturas de até aproximadamente $100{ }^{\circ} \mathrm{C}$, a quantidade de informações disponíveis sobre as propriedades térmicas de algumas espécies de madeira é relativamente ampla. No entanto, para temperaturas mais elevadas, isso não ocorre e estudos correlacionando as propriedades da madeira com a temperatura são relativamente escassos, ainda mais quando se trata de espécies de folhosas (dicotiledôneas), como é o caso do presente trabalho. $\mathrm{Na}$ maioria dos casos, os valores das propriedades da madeira são obtidos ou calibrados principalmente para espécies de coníferas.

Para facilitar o entendimento deste trabalho de pesquisa, serão descritos, inicialmente, alguns dos procedimentos experimentais realizados por Pinto (2005) para a análise de vigas de madeira em altas temperaturas. $\mathrm{O}$ trabalho experimental realizado por Pinto (2005) teve como objetivo a determinação da taxa de carbonização de um total de 6 vigas estruturais de madeira, de eucalipto citriodora, com seções transversais de dimensões $16 \times 26 \mathrm{~cm}$ e com $200 \mathrm{~cm}$ de comprimento. Para tanto, foram instalados nas vigas de madeira termopares do tipo $\mathrm{K}$, nas profundidades de 10, 20 e $30 \mathrm{~cm}$, totalizando 72 pontos de medida na seção considerada, (Figura 4). Durante os ensaios experimentais, as vigas foram expostas ao incêndiopadrão com aquecimento nas quatro faces, sendo que o aquecimento do forno de ensaios seguiu, neste caso, a curva ASTM E-119 (2000). Os ensaios experimentais foram realizados no forno vertical de ensaios do Laboratório de Desenvolvimento de Sistemas Construtivos de Furnas - Centrais Elétricas S.A., localizado em Aparecida de Goiânia - GO. Os termopares foram instalados ao longo de 3 pontos das vigas, ou seja, nas seções 1-3, (Figura 2), sendo que cada seção apresentou 12 pontos de leitura em 4 diferentes quadrantes, A, B, C e D, (Figura 3).

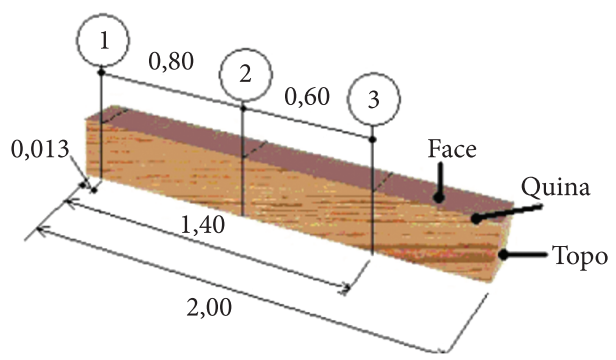

Figura 2. Seções 1-3 para implantação dos termopares. Fonte: Modificado de Pinto (2005)

Figure 2. Sections 1-3 for deployment of thermocouples. Source: Modified of Pinto (2005). 

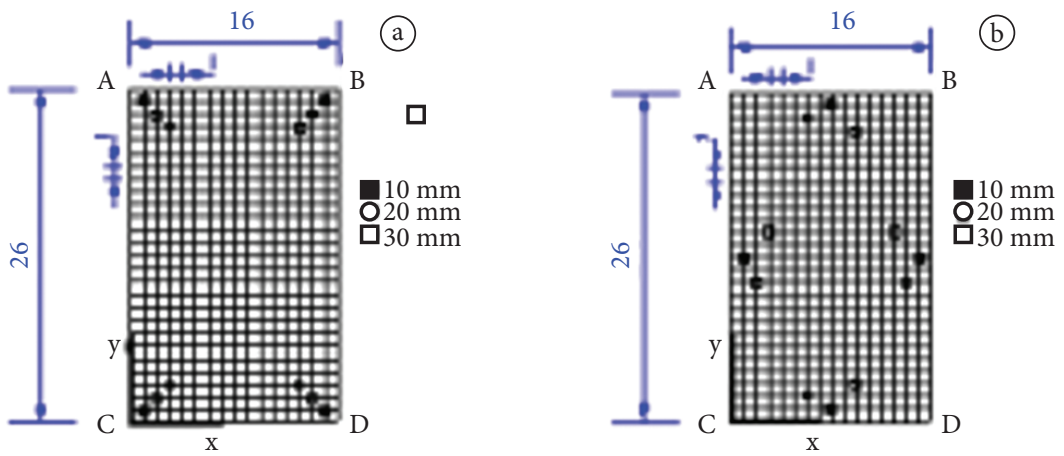

Figura 3. Posição dos termopares na análise experimental: a) Seções 1 e 3; b) Seção 2. Fonte: Pinto (2005).

Figure 3. Position of thermocouples in the experimental analysis: a) Sections 1 e 3; b) Section 2. Source: Pinto (2005).
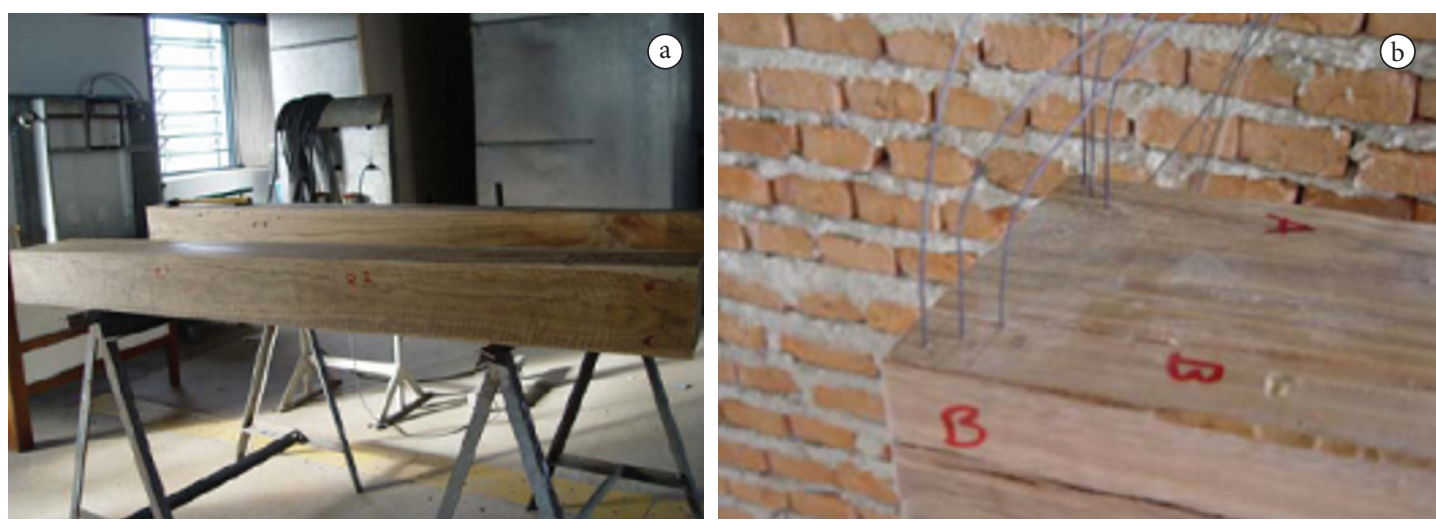

Figura 4. Vigas utilizadas na análise experimental: a) Preparação e marcação dos elementos; b) Fixação dos termopares. Fonte: Pinto (2005).

Figure 4. Beams used in the experimental analysis: a) Preparation and labeling of the elements; b) Attachment of thermocouples. Source: Pinto (2005).

Segundo Pinto (2005), as leituras obtidas para a seção 1 permitiram a avaliação da velocidade de carbonização no topo das peças de madeira, enquanto as leituras da seção 2 permitiram a avaliação da velocidade de carbonização das faces do elemento. Por fim, as leituras da seção 3 permitiram avaliar o efeito do arredondamento nas quinas da viga.

A temperatura adotada para a base carbonizada na análise experimental foi de $290^{\circ} \mathrm{C}$. Essa temperatura foi utilizada para avaliar o avanço da velocidade de carbonização da madeira, e é fundamental nos estudos sobre o processo de combustão da madeira.

Depois do fechamento do forno, uma vez as vigas no seu interior, o ensaio foi conduzido até o último termopar atingir a temperatura de $290{ }^{\circ} \mathrm{C}$, quando então os queimadores foram desligados, o forno aberto e as peças de madeira retiradas do interior do forno. A Figura 5 mostra a viga no interior do forno e após o ensaio com camada carbonizada.

Como resultado dos ensaios, Pinto (2005) relata que nos 10 minutos iniciais de exposição ao fogo ocorreu intensa liberação de fumaça e vapores de água, sendo que a liberação de fumaça precedeu uma súbita reação de combustão cuja temperatura foi marcada em $474{ }^{\circ} \mathrm{C}$. Porém, antes da reação de combustão, a temperatura do forno foi $19 \%$ maior que a temperatura da superfície da madeira, representando uma variação de aproximadamente $100{ }^{\circ} \mathrm{C}$. Depois da reação de combustão, ocorreu elevação da temperatura na superfície das vigas de modo que esta temperatura se manteve superior à temperatura do forno com formação de chamas e carvão incandescente na cor laranja, que se manteve até a finalização do ensaio. Pinto (2005) 

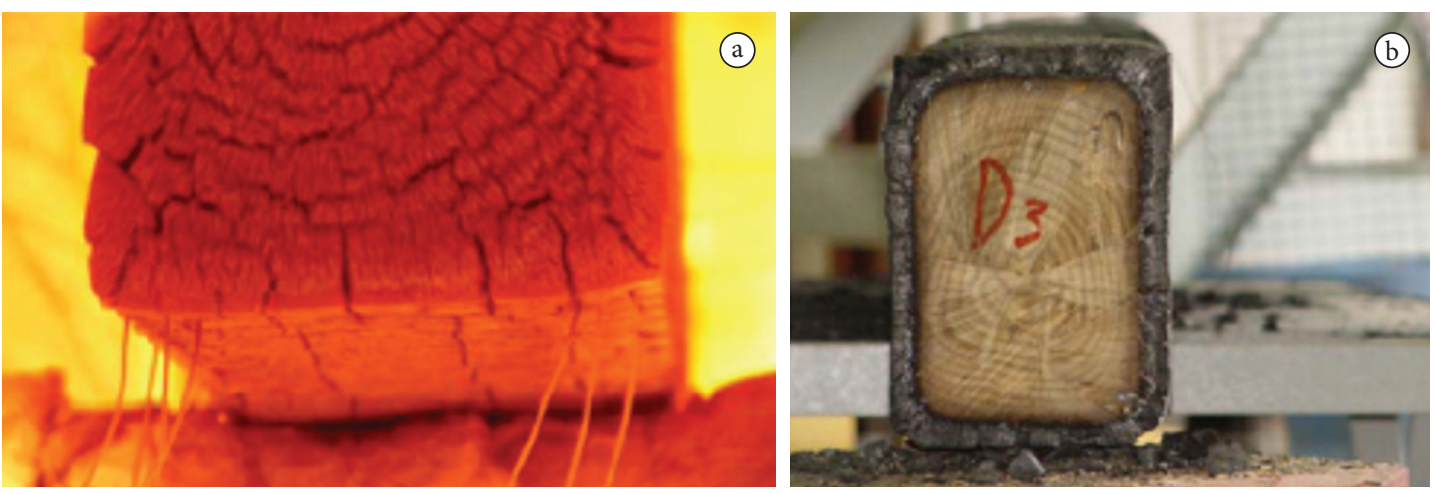

Figura 5. Análise experimental: a) Viga no interior do forno durante o processo de carbonização; b) Seção carbonizada após o ensaio. Fonte: Pinto (2005).

Figure 5. Experimental analysis: a) Beams into the furnace during charring process; b) Charred section after the test. Source: Pinto (2005).

relata ainda que, para valores próximos a $100{ }^{\circ} \mathrm{C}$, ocorreu interrupção na elevação da temperatura no interior da viga e consequente diminuição da taxa de carbonização, formando um patamar constante no gráfico Temperatura versus Tempo. Esse efeito termostático verificado próximo à temperatura de $100{ }^{\circ} \mathrm{C}$ tem relação com o ponto monitorado e a superfície da viga, ou seja, quanto mais superficial for o ponto de monitoração menor a permanência desse patamar. Além disso, a taxa de carbonização observada no topo da viga $(\beta=0,71 \mathrm{~mm} / \mathrm{min})$ foi ligeiramente superior à da quina $(\beta=0,73 \mathrm{~mm} / \mathrm{min})$, as quais foram superiores às da face $(\beta=0,58 \mathrm{~mm} /$ min). Os resultados permitiram observar que os valores experimentais para a taxa de carbonização da face: $0,58 \mathrm{~mm} / \mathrm{min}$ foram mais próximos dos valores tabelados pelo EUROCODE 5 (2002): $0,55 \mathrm{~mm} / \mathrm{min}$. Além disso, a maior dimensão da seção transversal da viga apresentou maior redução pela degradação térmica. Pinto (2005) apresentou também o gradiente térmico (Figura 6) com base nos resultados médios dos 72 termopares alocados na seção 2 para as 6 vigas consideradas na análise experimental, sendo as temperaturas expressas em cada centímetro. Deste modo, foi possível avaliar que, após aproximadamente 30 minutos de exposição ao fogo, com aquecimento padronizado pela curva ASTM E-119 (2000), a temperatura da superfície da madeira foi de $840^{\circ} \mathrm{C}$ e a $3 \mathrm{~cm}$ no interior da seção de madeira esta temperatura foi de $180^{\circ} \mathrm{C}$.

Além disso, ao final da exposição ao fogo, a redução da massa de madeira foi de aproximadamente $30 \%$

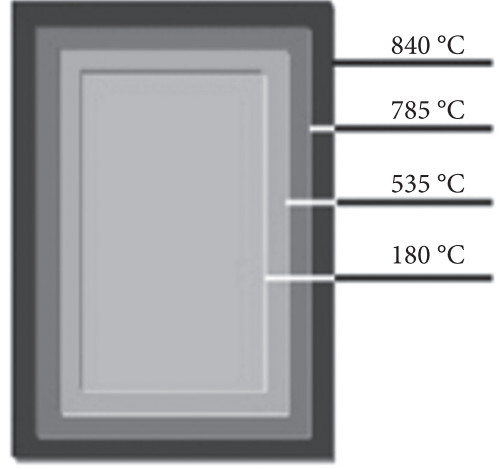

Figura 6. Gradiente de temperatura da seção correspondente a aproximadamente 30 minutos de exposição ao fogo para leituras da seção 2. Fonte: Pinto (2005).

Figure 6. Gradient of temperature in the section related to 30 minutes of fire to read of the section 2 . Source: Pinto (2005).

com relação à massa inicial. De uma maneira geral, com base nos ensaios experimentais analisados, a velocidade de carbonização da madeira apresentou um comportamento não linear, cuja velocidade foi maior para os milímetros iniciais e se reduziu com a profundidade, momento no qual assumiu um comportamento próximo à linearidade com menor variação nos dados, devido, principalmente, à formação de carvão, que atuou como isolante térmico na madeira.

O modelo numérico desenvolvido neste trabalho foi elaborado com base em elementos pré-definidos e disponibilizados na biblioteca interna do software 
ANSYS, versão 10.0. A opção pela utilização do ANSYS se deu pelo fato de que as ferramentas disponibilizadas pelo referido software permitem a avaliação da evolução da temperatura ao longo do tempo. A escolha de cada elemento finito foi feita a partir da consideração de sua representatividade perante o comportamento a ser simulado. Para a comparação dos resultados da modelagem numérica em questão, foram considerados os valores médios dos resultados experimentais obtidos para a seção 2 , (Figura 2), por Pinto (2005) e Regobello et al. (2006) para madeiras de eucalipto citriodora. A seção transversal do elemento de madeira com dimensões $6 \times 16 \mathrm{~cm}$ foi modelada por um elemento bidimensional do tipo plano (PLANE77) e por dois elementos do tipo superfície (SURF151) que foram utilizados para aplicar as condições de contorno de convecção e radiação ao modelo numérico, conforme recomendações de Regobello et al. (2006). O elemento PLANE77 apresenta oito nós com apenas um grau de liberdade por nó, ou seja, temperatura em cada nó. Já o elemento SURF151 pode ser usado para vários tipos de carregamento e efeitos de superfície em análises térmicas bidimensionais. $\mathrm{O}$ elemento SURF151 considera um nó específico (extra node), localizado fora da malha de elementos finitos, para controle da temperatura dos demais nós da malha, sendo que todos os nós são vinculados a ele. Os elementos PLANE77 e SURF151 são ilustrados, respectivamente, nas Figuras 7 e 8 .

A malha de elementos finitos referente à seção transversal da viga de madeira foi discretizada em elementos planos (PLANE77), tendo cada um dos elementos dimensões de $1,0 \times 1,0 \mathrm{~cm}$, totalizando 416 elementos (Figura 9). No perímetro da seção transversal referida, foram aplicados 96 elementos do tipo superfície (SURF151) em duas diferentes camadas, sendo 44 elementos destinados aos efeitos de radiação e os demais 44 elementos destinados aos efeitos da convecção na superfície do modelo considerado.

Para o modelo numérico, os valores utilizados para as propriedades térmicas da madeira em função da variação de temperatura foram calibrados por Regobello et al. (2006) para madeiras de eucalipto citriodora. Na Figura 10, estão apresentados os valores da condutividade térmica, sendo que o valor inicial adotado, neste caso, foi de $0,0020 \mathrm{~W} / \mathrm{cm} . \mathrm{K}$. A

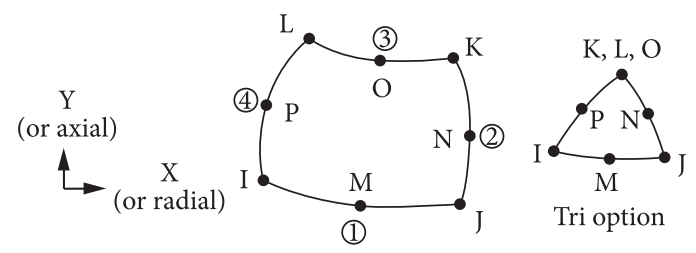

Figura 7. Elemento finito PLANE77. Fonte: Documentação do ANSYS.

Figure 7. Finite element PLANE77. Source: ANSYS documentation.

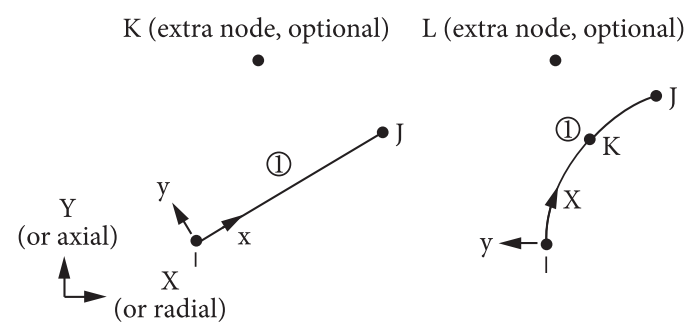

Figura 8. Elemento finito SURF151. Fonte: Documentação do ANSYS.

Figure 8. Finite element SURF151. Source: ANSYS documentation.

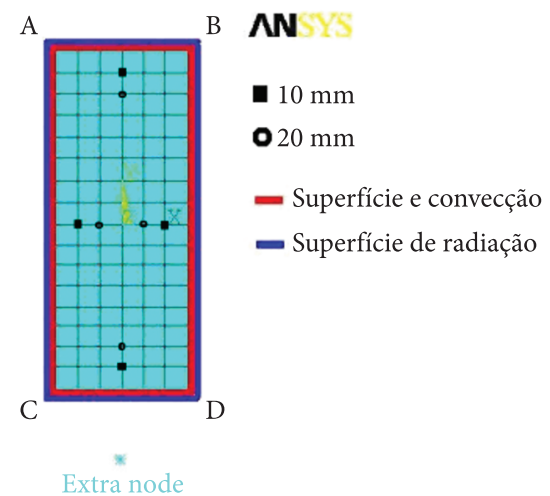

Figura 9. Malha de elementos finitos com representação das superfícies de convecção e radiação e profundidades de leitura das temperaturas no software ANSYS.

Figure 9. Mesh of finite elements with representation of surfaces of convection and radiation and depth readings of the temperatures in the software ANSYS.

variação dos valores do calor específico em função da temperatura está apresentada na Figura 11. O valor do coeficiente de convecção adotado para os elementos finitos (SURF151-convecção) que contornaram a superfície da seção transversal foi igual a $13,5 \times 10^{-4} \mathrm{~W} / \mathrm{cm}^{2} \mathrm{~K}$. O valor da emissividade adotado para os elementos finitos (SURF151radiação) que contornaram a superfície da seção 


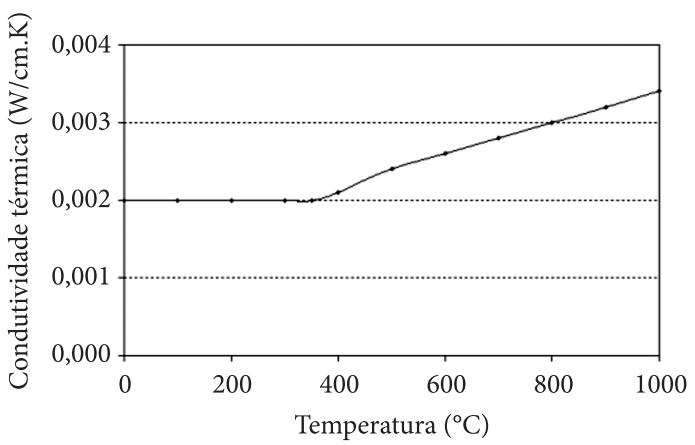

Figura 10. Curva da condutividade térmica em função da temperatura. Fonte: Modificado de Regobello et al. (2006).

Figure 10. Curves of thermal conductivity versus temperature. Source: Modified of Regobello et al. (2006)

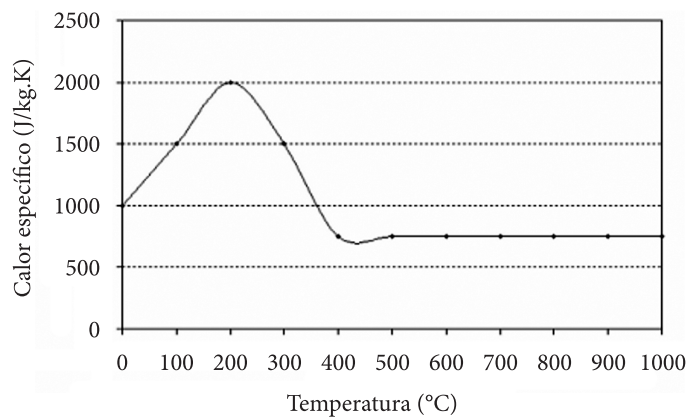

Figura 11. Curva do calor específico em função da temperatura. Fonte: Modificado de Regobello et al. (2006).

Figure 11. Curve of specific heat versus temperature. Source: Modified of Regobello et al. (2006).

transversal foi igual a 0,6. Segundo Jones (2001), tanto a emissividade quanto o coeficiente de convecção têm muito pouca influência no resultado do modelo numérico. Na Figura 12, estão apresentados os valores da variação da densidade da madeira em função da temperatura.

A análise considerada, neste caso, foi do tipo "térmica - transiente". Os resultados numéricos foram avaliados para o tempo $\mathrm{t}=30$ minutos (1800 segundos), sendo consideradas as profundidades de 10 e $20 \mathrm{~mm}$ na seção $6 \times 16 \mathrm{~cm}$ de madeira, (Figura 9).

\section{RESULTADOS E DISCUSSÃO}

As curvas de temperatura em função do tempo de exposição ao fogo, apresentadas na Figura 13,

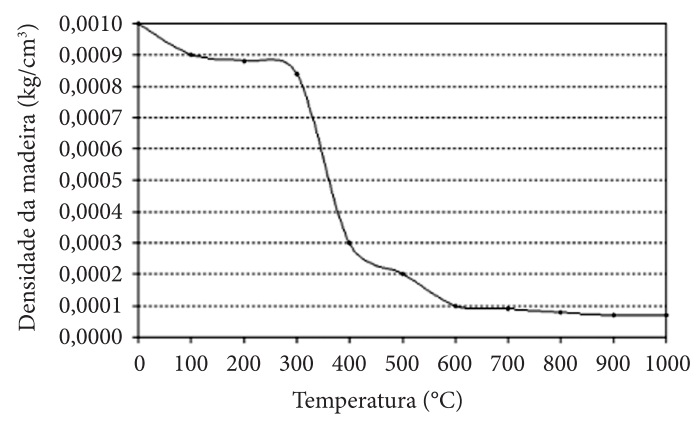

Figura 12. Curva da densidade em função da temperatura. Fonte: Modificado de Regobello et al. (2006).

Figure 12. Curve of density versus temperature. Source: Modified of Regobello et al. (2006)

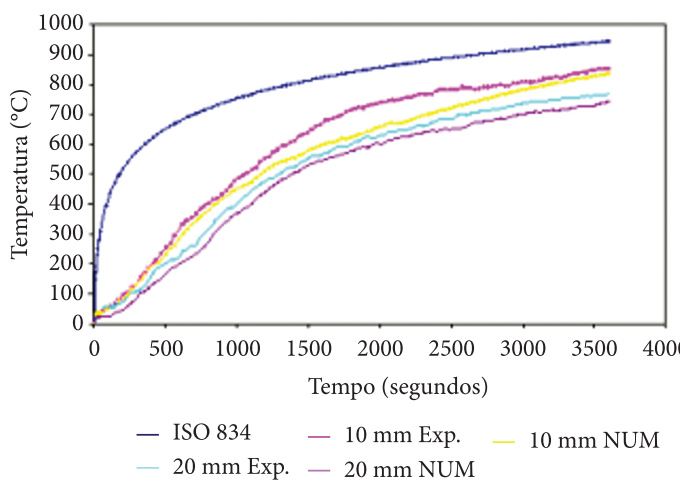

Figura 13. Comparação entre os resultados numéricos e experimentais (na seção 2 experimental) obtidos por Pinto (2005) para a evolução da temperatura em função do tempo.

Figure 13. Comparation between the numeric results and experimental (section 2) obtained by Pinto (2005) to evaluation of the temperature versus time.

foram obtidas com base na média dos valores obtidos para as quatro faces da Figura 9. Assim, por exemplo, para a profundidade de $10 \mathrm{~mm}$, a curva numérica apresentada corresponde à média dos valores de temperatura obtidos para a profundidade de $10 \mathrm{~mm}$ entre as faces $\mathrm{AB}, \mathrm{AC}, \mathrm{BD}$ e CD. A curva ISO 834 (1999) apresentada na Figura 9 corresponde ao controle de aquecimento do nó "extra node".

A partir da comparação entre os resultados apresentados na Figura 13, observou-se que os valores numéricos médios ficaram um pouco abaixo dos valores experimentais médios obtidos por Pinto (2005). 


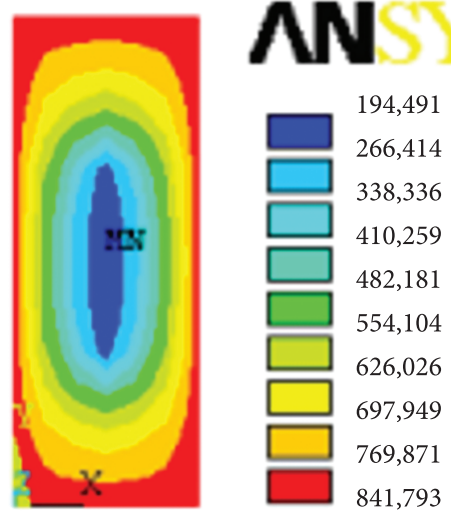

Figura 14. Campos de temperatura obtidos na análise numérica pelo software ANSYS para $\mathrm{t}=30$ minutos (1800 segundos).

Figure 14. Temperature obtained in the numerical analysis by software ANSYS to $\mathrm{t}=30$ minutes (1800 seconds).

Os valores obtidos para a profundidade de $20 \mathrm{~mm}$ foram mais próximos dos valores experimentais quando comparados com os valores obtidos para a profundidade de $10 \mathrm{~mm}$, aspecto provavelmente atribuído à falta de dados de condutividade para a espécie eucalipto citriodora estudada.

Além disso, os resultados numéricos obtidos para a seção $6 \times 16 \mathrm{~cm}$ foram próximos dos resultados obtidos por Regobello et al. (2006) para a seção $16 \times 26 \mathrm{~cm}$ de eucalipto citriodora para as profundidades 10 e $20 \mathrm{~mm}$.

A Figura 14 apresenta os campos de temperatura obtidos para o modelo numérico para um tempo de 30 minutos de exposição ao incêndio padrão de acordo com a ISO 834 (1999).

\section{CONCLUSÕES}

Os resultados do modelo numérico desenvolvido mostraram-se satisfatórios quando comparados aos resultados experimentais obtidos por Pinto (2005) e Regobello et al. (2006). O modelo aqui apresentado pode ser utilizado para a determinação da profundidade de carbonização da madeira com razoável aproximação. Além disso, para melhorar os resultados numéricos existe a necessidade da obtenção das propriedades térmicas para a espécie eucalipto citriodora, pois, com a inexistência das propriedades térmicas para folhosas, foram adotados valores experimentais de outros pesquisadores definidos para coníferas. Os valores das propriedades da madeira utilizados no presente trabalho servem como sugestão para futuros trabalhos relacionados à análise numérica em altas temperaturas. Há necessidade de se verificar o campo experimental de temperatura para outras dimensões de seção transversal e compará-los com resultados numéricos de seções de mesmas dimensões.

\section{AGRADECIMENTOS}

Agradecemos à Fundação de Amparo de Pesquisa do Estado de São Paulo (FAPESP) o apoio financeiro realizado.

\section{STATUS DA SUBMISSÃO}

Recebido: 25/08/2011

Aceito: 07/02/2012

Resumo publicado online: 22/03/2012

Artigo completo publicado: 30/06/2012

\section{AUTOR(ES) PARA CORRESPONDÊNCIA}

\section{Julio Cesar Molina}

Laboratório de Madeiras e de Estruturas de Madeiras - LaMEM,

Departamento de Engenharia de Estruturas - SET, Escola de Engenharia de São Carlos - EESC, Universidade de São Paulo - USP,

Av. Trabalhador São-carlense, 400, Centro, CEP 13560-970, São Carlos, SP, Brasil e-mail: juliocm@sc.usp.br

\section{REFERÊNCIAS}

American Society Testing and Materials - ASTM. ASTM-E119: standard test methods for fire testes of building construction and materials - Book of ASTM Standards. Philadelphia; 2000.

Associação Brasileira de Normas Técnicas - ABNT. NBR-7190: Projeto de Estruturas de Madeira. Rio de Janeiro; 1997.

Costa CN, Silva VP. Estruturas de concreto armado em situação de incêndio. In Anais da XXX Jornadas SulAmericanas de Engenharia Estrutural; 2002; Brasília. Distrito Federal: UNB; 2002, p 21. 
European Standard Eurocode. EUROCODE-5: Design of timber structures Part 1 - 2 - General rules - Structural Fire Design. Brussels: European Committee for Standardization; 2002.

International Organization for Standardization - ISO. ISO 834: Fire Resistance tests Part 1 - Elements of building construction - General requirements. Geneva; 1999.

Jones BH. Performance of Gypsium Plasterboard Assembies Exposed to Real Building Fires [dissertação]. Christchurch: University of Caterbury; 2001.
Pinto EM. Determinação de um modelo de taxa de carbonização transversal a grã para o eucalyptus citriodora e eucalyptus grandis [tese]. São Carlos: Universidade de São Paulo; 2005.

Regobello R, Pinto EM, Munaiar Neto J, Calil Junior C. Análise numérica e experimental da evolução de temperatura na madeira de eucalyptus exposta ao fogo. In: Anais do X Encontro Brasileiro em Madeira e em Estruturas de Madeira; 2006; São Pedro. São Paulo: Ebramem; 2006. p 15. 